\section{m/s}

médecine/sciences $1988 ; 4 ; 70-1$

\section{Pierre Freychet}

Professeur à la faculté de médecine, université de Nice

\section{ADRESSE}

P. Freychet: Inserm U. 145, faculté de médecine, 06034 Nice Cedex et service médecine interne n'3, hôpital de Cimiez,

\title{
MÉCANISME D'ACTION DE L'INSULINE
}

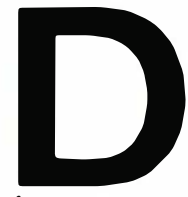

écouverte depuis plus de 40 ans, première hormone dont la séquence ait été établie et la synthèse chimique effectuée, parmi les premières hormones polypeptidiques pour la mise en évidence de récepteurs spécifiques, première hormone obtenue par ingéniérie génétique, l'insuline attend toujours l'élucidation de son mécanisme d'action. Situation paradoxale, eu égard aux recherches considérables effectuées dans ce domaine et à l'intérêt biomédical majeur que suscite cette hormone indispensable à la vie et aux grands équilibres métaboliques. Paradoxe seulement apparent, car la complexité et la multiplicité des effets de l'insuline rendent difficiles la formulation de concepts unicistes et l'élaboration d'approches capables de résoudre le problème d'une façon globale. L'insuline exerce deux grands types d'effets : métaboliques, et sur la croissance cellulaire [1]. Les effets métaboliques sont habituellement rapides (quelques secondes, ou minutes) : stimulation du transport et du métabolisme du glucose; inhibition de la lipolyse et de la glycogénolyse; inhibition de la néoglucogenèse. Les effets sur la croissance cellulaire sont plus lents (quelques heures, ou jours): stimulation de la synthèse globale des protéines, d'ARN et d'ADN. Cette distinction est cependant un peu artificielle. Certains effets métaboliques, comme la stimulation du transport des acides aminés, requièrent quelques heures, alors qu'un effet transcriptionnel spécifique (voir l'article de C. Dani et P. Grimaldi dans ce numéro, p. 90), tel que l'inhibition de la synthèse de l'ARNm de la phosphoénolpyruvate carboxykinase (PEPCK), est rapide (quelques minutes). Au niveau de la relation dose-effet, si les actions métaboliques classiques sont observées à basse concentration d'hormone (1 pmol/l à $1 \mathrm{nmol} / \mathrm{l}$ ) et les actions sur la croissance cellulaire à plus forte concentration, les effets transcriptionnels, plus récemment décrits, sont observés avec de très faibles concentrations d'insuline.

L'insuline, comme les autres hormones polypeptidiques, et les neuromédiateurs, se lie à un récepteur membranaire spécifique. Malgré de très nombreux travaux sur la liaison de l'insuline à son récepteur [1$3]$, on ne connaissait que peu de choses jusqu'en 1981 sur les mécanismes de l'action biologique de l'hormone. Depuis cette date, les renseignements accumulés sur la structure et la fonction du récepteur ont permis d'entrouvrir la «boîte noire » des étapes postérieures à la liaison de l'insuline à son récepteur [1, 3-6].

\section{RÉFÉRENCES}

1. Kahn CR. The molecular mechanism of insulin action Ann Rev Med 1985; 36 : 429-51.

2. Freychet $\mathbf{P}$. Interactions of polypeptide hormones with cell membrane specific receptors : studies with insulin and gluca gon. Diabetologia 1976; 12 : 83100.

3. Le Marchand-Brustel $Y$ Résistance à l'insuline dans l'obésité. médecine/sciences 1987 ; 3 : 394-402.

4. Gammeltoft S, Van Obberghen E. Protein kinase activity of the insulin receptor. Biochem J 1986 ; 235 : 1-11.

5. Czech MP. Molecular basis of insulin action(1 vol.). New York: Plenum Press, 1985.

6. Rosen OM. After insulin binds. Science 1987 ; 237 : 1452 8.

7. Houslay MD, Wakelam MJO, Pyne N J. The mediator is the message: is it part of the answer of insulin's action? Trends Biochem Sci 1986; 11 : 393-4.

$m / s n^{\circ} 2$ vol. 4, févier 88 
Le récepteur comporte deux sousunités glyco-protéiniques de 130 $(\alpha)$ et $95(\beta)$ kilodaltons. La structure primaire du récepteur humain a été déduite de la séquence de l'ADNc (voir l'article de E. Clauser dans ce numéro, p. 72) $[5,6]$. Alors que la sousunité $\alpha$ est exclusivement extracellulaire, la sous-unité $\beta$ comporte une séquence transmembranaire et, dans son domaine cytoplasmique, les éléments d'une tyrosine kinase. La sousunité $\beta$ présente des homologies de séquence avec le récepteur du facteur de croissance épidermique EGF, et avec les produits de certains oncogènes, en particulier $v$ ros. Le gène du récepteur humain est situé sur le bras court du chromosome 19. La sous-unité $\alpha$ lie l'insuline, ce qui conduit à l'autophosphorylation de la sousunité $\beta$, et produit l'activation de la tyrosine kinase de cette même sous-unité. Au moins trois ordres d'arguments suggèrent très fortement que la phosphorylation du récepteur et/ou l'activation de sa kinase sont impliquées dans la transmission du signal et les effets biologiques de l'hormone: tout d'abord l'insuline stimule, à des concentrations physiologiques et en moins d'une minute, la phosphorylation et l'activité tyrosine kinase de son récepteur, phénomène rapidement réversible [1]; ensuite, l'activité kinase du récepteur varie parallèlement à l'action de l'insuline dans des états de résistance ou de réponse accrue à l'hormone $[1,3]$; enfin, l'expression, par une lignée cellulaire, du récepteur humain entier, muté, ou tronqué, a permis de préciser le fonctionnement du récepteur et le rôle de sa kinase dans les effets biologiques de l'insuline (voir l'article de E. Clauser dans ce numéro). La structure et les fonctions du récepteur sont pratiquement identiques dans toutes les cellules et espèces étudiées jusqu'ici, bien qu'un certain degré de micro-hétérogénéité dans la structure ait été observé $[1,4]$.

$\mathrm{m} / \mathrm{s} n^{\circ} 2$ vol. 4 , feurier 88
L'action de l'insuline sur des cellules intactes se traduit, en particulier, par la phosphorylation/déphosphorylation sur des résidus sérine/thréonine de nombreuses protéines, dont plusieurs enzymes [5]. Une (ou plusieurs) sérine kinase(s), étroitement associée(s) au récepteur sans en être partie intégrante, pourrai(en)t être impliquée(s) dans la transmission du signal entre la tyrosine kinase du récepteur et la cascade de phosphorylation/déphosphorylation subséquente [4]. De nombreux travaux se sont attachés à la découverte d'un «second messager » de l'action de l'insuline [1, 5-7]. La plupart des messagers supposés (modifications des contenus en AMPc et GMPc; flux ioniques et altérations du potentiel de membrane; mouvements de calcium; peptides provenant de la membrane plasmique) n'ont pas été confirmés. Des travaux récents ont attiré l'attention sur un nouveau médiateur glycolipidique $[6,7]$ dont une partie de la structure est semblable à celle des phosphoinositol glycans qui servent d'ancrage membranaire à certaines protéines extracellulaires. Une autre acquisition importante des dernières années concerne la stimulation du transport du glucose par l'insuline, dont l'effet est de provoquer un déplacement des transporteurs à partir d'un compartiment intracellulaire vers la membrane plasmique $[1,5]$.

Comme beaucoup d'autres ligands, l'insuline liée à son récepteur subit une internalisation par endocytose qui semble intervenir surtout dans la dégradation de l'hormone (voir l'article de J.L.Carpentier dans ce numéro, p. 83). Le transfert intracellulaire des récepteurs participe également, avec leur synthèse de novo et leur recyclage vers la membrane plasmique, au contrôle du nombre des récepteurs disponibles à la surface cellulaire et, ainsi, à la modulation de la sensibilité des cellules cibles à l'hormone. Il est possible que l'internalisation du récepteur permette d'amener sa kinase activée au contact de substrats intracellulaires.

De nombreux travaux ont été récemment consacrés à la recherche de substrats endogènes pour la tyrosine kinase du récepteur. Ils n'ont rencontré, jusqu'ici, qu'un succès limité $[4,6]$. Plusieurs protéines sont des candidats possibles, mais leur identité et leur rôle biologique restent à élucider. En fait, le seul substrat clairement identifié à ce jour est la sous-unité $\beta$, et il est possible que le récepteur lui-même soit le principal substrat pour sa propre activité kinasique. L'autophosphorylation de la sous-unité $\beta$ pourrait affecter une fonction encore inconnue du récepteur, ou permettre à celui-ci de modifier de façon non covalente d'autres protéines membranaires ou cytosoliques, modifications génératrices d'un signal impliqué dans les effets biologiques.

Une anomalie quantitative (diminution de la synthèse) ou qualitative (altération de l'affinité) du récepteur, probablement génétique, a été décrite dans certains syndromes, rares, d'insulino-résistance sévère ; une altération localisée à l'activité kinase du récepteur a été rapportée chez certains de ces patients $[1,6]$. Il est désormais possible de rechercher des altérations au niveau du gène du récepteur humain. Parallèlement, les progrès concernant, en particulier, le rôle de la phosphorylation du récepteur et/ou de son activité tyrosine kinase dans la transmission du signal, devraient contribuer à l'élucidation du mécanisme d'action. Ils devraient aussi permettre d'élaborer de nouvelles stratégies pour le traitement et la prévention de l'insulinorésistance, fréquente dans les diabètes où elle joue un rôle important dans la physiopathologie de la détérioration métabolique, et dans la pathogénie des complications de la maladie 